\title{
Cardiac and vascular point-of-care ultrasound: current situation, problems, and future prospects
}

\author{
Hirotsugu Yamada ${ }^{1,2}\left(\mathbb{0} \cdot\right.$ Hiroyuki Ito $^{1,2} \cdot$ Mika Fujiwara $^{2}$
}

Received: 16 August 2021 / Accepted: 21 September 2021 / Published online: 8 January 2022

(c) The Author(s), under exclusive licence to The Japan Society of Ultrasonics in Medicine 2021

\begin{abstract}
Although clinical application of ultrasound to the heart has a history of about 80 years, its big turning point was the emergence of a portable ultrasound diagnostic machine. As a result, the place, where echocardiography is performed widely spread outside the examination room, and the people who perform echocardiography have also greatly increased. Emergency physicians, anesthesiologists, and primary care physicians became interested in echocardiography and started using it. Such ultrasound examinations performed by a doctor for assessment of disease condition, management, or guidance of treatment at bedside has been called point-of-care ultrasound (POCUS). Cardiac POCUS is divided into a focused cardiac ultrasound examination (FoCUS) and limited echocardiography. The former is performed by non-experts in echocardiography, such as emergency physicians and anesthesiologists, whereas the latter is usually performed by cardiologists who are experts in echocardiography. FoCUS has an established protocol and evaluation method, and evidence to prove its effectiveness is accumulating. In addition, the COVID-19 outbreak reaffirmed the importance of POCUS. Although FoCUS is becoming popular in Japan, an educational program has not been established, and discussion on how to educate medical students and residents will be necessary. Even if POCUS in cardiovascular medicine becomes widespread, auscultation will still be necessary. Rather, adding cardiac and vascular POCUS to inspection, palpation, and auscultation in the flow of physical examinations will benefit patients greatly.
\end{abstract}

Keywords Point-of-care ultrasound · Focused cardiac ultrasound · Limited echocardiography $\cdot$ Portable ultrasound diagnostic machine $\cdot$ Vascular ultrasonography

\section{Introduction}

Ultrasound in the cardiovascular setting had been performed only by doctors specializing in cardiovascular medicine in the early days of its clinical application. The ultrasound examination has the advantage that it can be performed outside the laboratory, which is not possible with other diagnostic imaging modalities such as computed tomography and magnetic resonance imaging. With the development of

Hirotsugu Yamada

yamadah@tokushima-u.ac.jp

1 Department of Community Medicine for Cardiology, Tokushima Graduate School of Biomedical Sciences, 2-50-1 Kuramoto, Tokushima 770-8503, Japan

2 Department of Cardiovascular Medicine, Takamatsu Municipal Hospital, Takamatsu, Japan a small portable ultrasound diagnostic device, this advantage was further emphasized. Then, doctors in various clinical departments have come to utilize cardiovascular ultrasound examination in a different way. In this paper, we would like to look back on the history leading up to this issue, summarize the current situation, and think about future prospects.

\section{History of echocardiography}

The clinical application of ultrasound imaging to the heart began in the 1950s with the study of A-mode and M-mode echocardiography by Edler and Herz [1]. Tanaka et al.[2] subsequently developed heartbeat-synchronized tomography in 1964, and Matsuo et al. developed electronic scanning ultrasound tomography in 1976. Two-dimensional echocardiography then came to be applied clinically, and clinical echocardiography was pioneered by Feigenbaum [3], who is called 
the father of echocardiography, and the Mayo Clinic group. Furthermore, the color Doppler method was developed by Namekawa et al. in the 1980s [4]. Since then, the ultrasound examination has played a leading role in morphological and functional diagnosis of cardiac disease, and "echocardiography" has been established as one of the major academic fields of cardiology. Against this background, echocardiography has been regarded as an examination that requires highly specialized, complicated, and expensive equipment. Originally, to perform echocardiography at the bedside of the ward, emergency outpatient department, or intensive care unit, a heavy machine was moved from the examination room and the examination was performed by a cardiologist. Later, the number of facilities deploying abdominal ultrasound examination devices in the emergency department or intensive care unit increased. As many of the ultrasound diagnostic equipment gradually became general-purpose machines, echocardiography was performed even with the equipment deployed in the emergency outpatient department and intensive care unit. This process has increased the chances of emergency physicians and intensive care physicians performing echocardiography.

\section{History of vascular ultrasound examination}

The application of vascular ultrasonography has begun to be applied to the aorta as part of echocardiography or abdominal ultrasound examination. Around 1980, in addition to the diagnosis of abdominal aortic aneurysm or dissection, evaluation of obstructive lesions in peripheral arteries began using the Duplex ultrasound system (B-mode and color Doppler techniques). From a similar time, evaluation of venous disease in lower extremities began to be performed at specialized facilities. Since 1980, with advances in ultrasound diagnostic equipment, the observation range has expanded to the carotid artery, peripheral arteries and veins, renal arteries, and so on. In addition, vascular ultrasound has come to be frequently used as a guide for procedures, such as ultrasound-guided vascular puncture and vascular access. In 2017, the Japan Society of Ultrasonics in Medicine published "Standard method for ultrasound evaluation of deep vein thrombosis and lower extremity varicose veins" [5]. This guideline completed the series of Japanese guidelines for vascular ultrasound, accelerating the spread of vascular ultrasonography all over the country.

\section{Diversification of cardiovascular ultrasonography due to miniaturization of ultrasound examination devices}

In 1998, the battery-powered lightweight portable ultrasound diagnostic device "SonoSite 180" was developed with a grant from the United States Department of Defense, and it was put on the market. In 2000, the "Sonoheart" for echocardiography was launched in the same series, and the "SonoSite 180 PLUS" equipped with the Doppler mode was launched in 2002. Other companies have followed, and various portable devices are now on the market [6]. The "Vscan", marketed by GE Healthcare in 2010, is a lightweight ultrasound diagnostic device that weighs $390 \mathrm{~g}$ and fits in the pocket. With the commercialization of such devices, not only in-hospital intensive care units and emergency outpatient departments but also various places, such as disaster and accident sites, doctor cars and helicopters, home medical care, sports venues, battlefields, space stations, and so on became a place to perform ultrasound diagnosis. Most of the ultrasound examinations performed in such situations are point-of-care ultrasound (POCUS). The development and marketing of portable ultrasound diagnostic equipment and the spread of POCUS are closely related. In this way, such as echocardiography, high-performance compact ultrasound diagnostic equipment will bring a major change to the utilization of ultrasound examinations in all medical areas.

As a result of the fact that echocardiography can be performed at the bedside, not only echocardiographic specialists but also non-echocardiographic specialists such as general physicians, emergency doctors, and anesthesiologists can perform the examination. Echocardiography by non-specialists has come to be used as a tool to obtain clinical answers with limited goals such as whether there is heart failure and whether there is fluid retention, and so on. The use of such echocardiography has spread in the fields of emergency medical care and intensive medical care, and it has become commonplace to use an ultrasound device for evaluation of hemodynamics [7-13]. In addition, with the spread of vascular ultrasound, it has come to be performed in the emergency and intensive care areas. In particular, the diagnosis of deep vein thrombosis with lower limb vein ultrasonography using a portable ultrasound device in a disaster area has been in the spotlight [14].

\section{Popularization of POCUS}

With the development of high-performance compact ultrasound diagnostic equipment, doctors other than ultrasound specialists have more opportunities to perform ultrasound examinations at the bedside. Such ultrasound examinations have been called by various names (Table 1). All are ultrasound examinations performed by a doctor on the patient in front of him or her to evaluate the patient's condition or guide the procedure [15]. In 2011, a review paper entitled "point-of-care ultrasonography" was published in the New England Journal of Medicine, and point-of-care ultrasound (POCUS) became well known [16]. In 2016, the first 
meeting of the Society of Point-of-Care Ultrasound was held in Japan, as well. POCUS targets not only the heart but also organs throughout the body.

\section{Simple protocol for cardiac ultrasound examination}

With the widespread use of such small portable devices, a wide variety of medical professionals who are not specialists in echocardiography have begun to perform cardiac ultrasound examinations in various situations [17]. For nonspecialist medical staff to properly perform the examination, a simple protocol that can be acquired with a short training period was devised. Some proposed protocols using ultrasound including cardiac ultrasound are listed in Table 2. The cardiopulmonary limited ultrasound examination (CLUE) proposed by Kimura et al. is an examination

Table 1 Terms in use that may refer to FoCUS [17]

Table 2 Protocols for cardiac point-of-care ultrasound \begin{tabular}{l} 
Hand-held cardiac ultrasound \\
Point-of-care cardiac ultrasound \\
Ultrasound stethoscope \\
Hand-carried cardiac ultrasound \\
Bedside cardiac ultrasound \\
Quick-look cardiac ultrasound \\
\hline
\end{tabular}

FoCUS focused cardiac ultrasound protocol performed by primary care physicians in outpatient clinics and is a combination of echocardiography and lung ultrasound examination [18]. In addition, the focused echocardiographic evaluation in life support (FEEL) protocol [8, 19] was devised mainly by anesthesiologists, and the focus assessed transthoracic echocardiography (FATE) protocol was developed mainly by emergency physicians, and each training course has been held [20,21]. Guidelines for echocardiography in emergency medicine [22-24] and intensive medical care $[22,25]$ were subsequently developed. Under these circumstances, guidelines for focused cardiac ultrasound examination (FoCUS) [23] was published one after another by the American Society of Echocardiography in 2013, and guidelines for focused cardiac ultrasound examination were published by the European Society for Cardiovascular Imaging in 2014 [26]. In the same year, a guideline summarizing international evidence was also published [27], and cardiac POCUS has come to be popularly known as FoCUS. The FoCUS protocol uses three approaches: the subcostal approach, the left parasternal approach, and the apical approach, and mainly observes five cross-sectional images (Fig. 1).

In fact, the United States and Europe have a slightly different way of thinking about FoCUS, with the United States guidelines limiting the tests to be performed by non-specialists using a portable ultrasound diagnostic device and assuming that detailed evaluation of heart disease is not possible. On the other hand, European guidelines are mainly intended

\begin{tabular}{|c|c|c|}
\hline Protocol & Targets of the observation & Observation views \\
\hline CLUE [18] & $\begin{array}{l}\text { LV systolic dysfunction } \\
\text { LA enlargement } \\
\text { IVC plethora } \\
\text { Ultrasound lung comet-tail artifacts (ULC) }\end{array}$ & $\begin{array}{l}\text { Parasternal long-axis view } \\
\text { Longitudinal subcostal view } \\
\text { Lung apical views }\end{array}$ \\
\hline FATE $[8,19]$ & $\begin{array}{l}\text { Pericardial effusion } \\
\text { Dilated RA, RV } \\
\text { Dilated LA, LV } \\
\text { LV hypertrophy } \\
\text { LV, RV systolic function } \\
\text { Pleural effusion }\end{array}$ & $\begin{array}{l}\text { Subcostal 4-chamber view } \\
\text { Apical 4-chamber view } \\
\text { Parasternal long-axis view } \\
\text { Parasternal short-axis view } \\
\text { Bilateral pleural scans }\end{array}$ \\
\hline FEEL $[20,21]$ & $\begin{array}{l}\text { Pericardial effusion } \\
\text { LV systolic function } \\
\text { Pleural effusion }\end{array}$ & $\begin{array}{l}\text { Subcostal 4-chamber } \\
\text { Subcostal long-axis } \\
\text { Parasternal short-axis } \\
\text { Parasternal long-axis } \\
\text { Apical 4-chamber view } \\
\text { Pleura }\end{array}$ \\
\hline FoCUS $[17,27]$ & $\begin{array}{l}\text { LV dimension, systolic function } \\
\text { RV systolic function } \\
\text { Volume status } \\
\text { Pericardial effusion, tamponade physiology } \\
\text { Gross signs of chronic heart disease } \\
\text { Gross valvular abnormalities } \\
\text { Large intracardiac masses }\end{array}$ & $\begin{array}{l}\text { Subcostal long-axis view } \\
\text { Subcostal inferior vena cava view } \\
\text { Parasternal long-axis view } \\
\text { Parasternal short-axis view } \\
\text { Apical 4-chamber view }\end{array}$ \\
\hline
\end{tabular}

CLUE cardiopulmonary limited ultrasound examination, FATE focus assessed transthoracic echo, FEEL focused echocardiographic evaluation in life support, FoCUS focused cardiac ultrasound examination 
for use in emergency settings and assume these examinations will be performed by those with the same level of echocardiographic knowledge and skills as cardiologists to achieve high-quality patient care (Table 3 ). The observation views



Fig. 1 Basic observation approaches and views for focused cardiac ultrasound examination on FoCUS are almost the same in the European and United States guidelines. However, it seems that Europe places more importance on the supervision of FoCUS and its quality control by an accredited echocardiography laboratory or emergency department.

\section{FoCUS and other echocardiography}

The guidelines issued by the American Society of Echocardiography in 2013 distinguish FoCUS from echocardiography [23]. Conventional echocardiography is called "comprehensive echocardiography" or "complete echocardiography" and is systematic or comprehensive echocardiography. FoCUS is a test that can be performed with a short training period even by medical professionals who have little knowledge or experience of specialized echocardiography, and it is desirable that the technique be maintained with daily use. On the other hand, to perform comprehensive echocardiography, a wide range of advanced knowledge from basic ultrasound engineering to pathophysiology and treatment of various cardiac diseases is required. It is necessary to measure various cardiac functions by making full use of many echocardiographic techniques. For comprehensive echocardiography, a high-end machine equipped with all options is desirable, but FoCUS can also be performed with a portable device with limited capability. Of course, if you have the equipment available at the bedside, FoCUS can be performed with a high-end machine.

On the other hand, echocardiography specialists have traditionally performed bedside echocardiography, which is called "limited echocardiography". Limited echocardiography is a bedside echocardiography performed by someone with advanced knowledge and skills in systematic echocardiography. This is also a form of cardiac POCUS, but not FoCUS. Ultrasound guidelines make a

Table 3 Summary of the European Association of Cardiovascular Imaging (EACVI) viewpoint on FoCUS [28]

FoCUS should only be used as a point-of-care cardiac ultrasound examination, aimed to detect a limited number of critical cardiac conditions

FoCUS may provide key clinical information regarding the presence of pericardial effusion/cardiac tamponade, left and right ventricular size and function, intravascular volume status, and may aid decision-making during cardiopulmonary resuscitation

FoCUS should never be considered or reported as echocardiographic examination

Educational curriculum and training programme for FoCUS should be designed and conducted by the specialty professional organizations/societies involved in treating medical emergencies, including cardiac, with continual collaboration with reference echocardiographic communities

FoCUS should only be used by the operators who have completed appropriate education and training programme, and who fully understand and respect its scope and limitations

Whenever the information about cardiovascular abnormalities provided by the FoCUS exam is insufficient for the immediate or definitive care of patients, these should be referred to a comprehensive echocardiographic examination as soon as possible, and as compatible with clinical priorities

FoCUS examinations should be recorded and permanently stored and reports issued in a timely manner

Continual supervision and quality control of the FoCUS examinations are essential, provided preferably by accredited echocardiographic laboratories and emergency echocardiography services

Reference echocardiographic community representatives should actively follow developments in the field and, whenever appropriate, work on improving educational and training curricula in concert with respective specialties professional societies/organizations, to deliver the best possible care for the patients 
clear distinction between this limited echocardiography and FoCUS (Table 4). In the United States, for example, limited echocardiography is covered by health insurance, but FoCUS is not. Under the Japanese health insurance system, ultrasonography is not diagnostic imaging including interpretation and is treated the same as a blood test. Although even FoCUS may be covered by insurance as ultrasonography, many Japanese medical institutions do not submit a claim for FoCUS and limited echocardiography.

In this way, FoCUS may be considered different from the conventional comprehensive and limited echocardiography, even if they are all ultrasound examinations. However, they are complementary to each other. In other words, if any abnormality in the heart is detected with FoCUS, it will be necessary to perform follow-up comprehensive echocardiography to diagnose the pathological condition. In addition, even if there is no abnormality detected on FoCUS, if cardiac disease is suspected from symptoms or other clinical findings, it will be necessary to repeat FoCUS after a while, but at some point limited or comprehensive echocardiography is recommended. It would be dangerous to conclude that there are no cardiac abnormalities, because there are no abnormal findings detected on FoCUS [28].

Table 4 Differences between limited echocardiography and FoCUS [17]

\footnotetext{
Limited echocardiography

Definitive examination that requires knowledge and expertise described below

Knowledge that specific additional images would be useful

Expertise to acquire additional images from all acoustic windows

Knowledge that a specific additional ultrasound technique would be useful

Expertise to acquire additional images with all cardiac ultrasound imaging modalities

Knowledge to identify all expected normal structures and/or artifacts from all views

Knowledge to identify pathologic findings on structures of clinical interest

Knowledge to look for and identify lesions associated with other findings, whether in the same view of other parts of the study

Knowledge to identify incidental findings within images acquired

Knowledge of quantitative techniques

Expertise to apply quantitative techniques

Expertise to answer any referral question with appropriate negative and positive pertinent findings

FoCUS

Identify the presence or absence of one or several specific findings using a defined, preestablished image acquisition protocol
}

FoCUS focused cardiac ultrasound

\section{Protocol for cardiac POCUS}

If we try to obtain a lot of information from one examination, we will increase the number of observed imaging views, add the color Doppler method, and complicate the inspection procedure. Since it is assumed that FoCUS will be performed by a non-specialist, if the number of evaluations and observations is increased too much, it will be complicated, and it will be difficult to acquire and maintain the technique, because it is not suitable for emergency situations. On the contrary, if the number of views is reduced and the inspection procedure is simplified, the amount of information that can be obtained naturally decreases. The balance point may differ depending on the position of the doctor who needs the examination and what the doctor wants.

For example, if you are a primary care physician diagnosing a chronic disease, the minimum necessary information will be very useful, and an examination with a simple procedure such as the CLUE protocol described above will suffice. On the other hand, if you are an emergency doctor who deals with patients who are transported to the emergency department, you should expand the procedure a little more and include the FATE exam or echocardiography as part of the RUSH exam. A cardiologist called after seeing an emergency doctor in the emergency department evaluates abnormal hemodynamics by making full use of the Doppler method and decides the treatment strategy. Of course, whether you are a primary physician or an emergency physician, it is better if you have the skills of a cardiologist who can perform echocardiography. In addition, the latest ultrasound diagnostic equipment, even if it is a standard machine, has almost the same functions and capabilities as high-end machines in terms of diagnosing cardiac diseases. Therefore, even a non-specialist can significantly shorten the time to diagnosis if he or she has skills similar to those of an expert in echocardiography.

\section{Vascular POCUS}

It is well known that the ultrasound-guided method improves the success rate and reduces complications when placing a central venous catheter $[29,30]$ and is widely used. Therefore, it can be said that many doctors are proficient in observing blood vessels by ultrasound device. The RASH examination in the emergency setting includes observation of the thoracoabdominal aorta and the proximal deep veins in addition to the inferior vena cava. It has been reported that the " $4 \mathrm{~S}$ " approach is useful for visualizing the aorta. In the method, one observes the 
ascending aorta in the superior sternal view, descending aorta in the small-scale view, abdominal aorta in the subxiphoid view, and aortic arch in the supra-sternal view [31]. In ultrasound examinations of lower extremity veins for diagnosing deep vein thrombosis, the 2-point compression test, which observes the common femoral vein and the popliteal vein by the compression method, is useful in the emergency setting [32-34]. The compression method has been widely used by emergency physicians. In the recently published "Standard method for ultrasound evaluation of deep vein thrombosis and lower extremity varicose veins" by the Japan Society of Ultrasonics in Medicine, it is recommended to perform a 2-point compression test in the acute phase in high-risk cases. For the aorta, it is almost impossible to search the entire area of the aorta by ultrasound examination, and the gold standard for diagnosing aortic dissection is contrast-enhanced CT. The 2-point compression test cannot diagnose thrombosis in the pelvic and lower legs. Thus, to make effective use of POCUS, it is necessary to always be aware of what can be observed and what is not seen.

\section{Application of POCUS during echocardiography}

Another way to use POCUS is to perform additional POCUS during echocardiography in the laboratory. For example, when pulmonary hypertension is diagnosed with echocardiography and the cause is thought to be pulmonary embolism, it is necessary to check for deep vein thrombosis. If one performs the above 2-point compression test immediately after echocardiography, it can be diagnosed immediately. In addition, when the cause of chest pain is investigated and echocardiography is performed and there is no cause in the heart, lung POCUS can be performed to diagnose pneumothorax, or vascular POCUS can be performed to diagnose aortic dissection. In facilities, where the examination is performed only by the technician, the extent to which the technician is allowed to expand the examination scope may be an issue, but if the examination is performed by the doctor, a diagnostic approach using POCUS is very useful for clinical decision making.

\section{POCUS education in the cardiovascular setting}

With the introduction of new technologies and measurement methods, echocardiography has become complicated and a very difficult examination for beginners to perform. Sonographers perform echocardiography in hospitals, where many residents start their clinical training, and this has decreased the opportunity for them to train in echocardiography. The situation is much the same for cardiology residents. They often work in the catheter laboratory, but not in the ultrasound examination laboratory. As a result, many young physicians think that echocardiography is an examination performed by a technician. Under these circumstances, FoCUS may also play a role in lowering the hurdle for echocardiography. FoCUS is a protocol developed by non-cardiologists for non-cardiologists, but it is also a useful technique for early residents and young doctors aiming to become cardiologists. We would like to propose a new method for learning echocardiography for doctors, starting with FoCUS to get used to echocardiography, gradually deepening knowledge and skills, and ultimately becoming able to perform comprehensive echocardiography.

\section{Will portable ultrasound devices replace the stethoscope?}

The time has come, where observing organs with an ultrasound device becomes part of a physical examination, similar to listening to heart sounds and lung sounds with a stethoscope [35]. The radical opinion that cardiologists should quit auscultation and look at echoes may be a good slogan for the spread of ultrasound examination [36, 37], but of course, stethoscopes are never useless. In addition to inspection, palpation, and auscultation in the course of a physical examination, adding ultrasound to the mix will bring great benefits to patients.

To bring about such an era, it will be necessary to introduce ultrasound from the early stage of medical education. Currently, the goal set by the Ministry of Health, Labor and Welfare for initial residents is to be able to perform ultrasound examinations and interpret the results, but the specific details have not been specified in Japan. In the future, it will be necessary to set the required items and goals for each area by ultrasound examination. In addition, in pre-graduate education, attempts are being made to issue pocket ultrasound devices to medical students in the United States. Currently, Japanese medical students who have passed the Computer Based Testing (CBT) and Objective Structured Clinical Examination (OSCE) are awarded the Student Doctor Certificate of the National Medical School Directors' Meeting and their university. We hope that the practical skills of ultrasonography will be incorporated into the OSCE in the near future. For that purpose, deploying an ultrasound diagnostic device that can be used by medical students during the day at the university and training instructors of ultrasound will be indispensable. 


\section{POCUS in the COVID-19 era}

POCUS has been increasingly used for the detection of not only lung lesions but also cardiovascular complications of coronavirus disease 2019 (COVID-19) [38]. In patients with COVID-19, cardiac POCUS is recommended, because it has similar benefits to transthoracic echocardiography while decreasing staff exposure to patients with COVID-19 and the risk of infection. Handheld or smaller lap-top ultrasound devices have an advantage as they are easier to cover, clean, and disinfect than larger standard machines with higher capability [39]. The American Society of Echocardiography (ASE) recommends using POCUS as a first-line examination in confirmed and suspected COVID-19 patients [40, 41]. Up to $50 \%$ of patients with COVID-19 have cardiac abnormalities, such as myocardial injury, myocarditis, cardiac arrythmia, venous thromboembolism, and acute coronary syndrome [42]. These complications lead to management changes in nearly one-third of such patients [43]. During the COVID-19 pandemic, among various other imaging modality, it is necessary to improve the prognosis of patients by appropriate use of POCUS while preventing the spread of infection [44].

\section{Conclusion}

The current status of cardiovascular POCUS was outlined, and associated problems and future prospects were described. As you can see from the special features in this journal, ultrasonography has been traditionally developed independently in each area (organ) in clinical medicine, as well as in research and education. However, the authors think that there is no organ-specific POCUS in the emergency setting and primary care setting. To popularize and teach POCUS, the first step is to be aware that POCUS needs to be used across regions, rather than being performed on an organ-by-organ basis as in conventional ultrasonography.

\section{Declarations}

Conflict of interest The authors declare that there are no conflicts of interest.

Ethical statements There are no ethical issues to disclose (the article is not original research).

\section{References}

1. Edler I, Gustafson A. Ultrasonic cardiogram in mitral stenosis: preliminary communication. Acta Med Scandinav. 1957;159:85-90.

2. Ebina T, Oka S, Tanaka M, et al. The ultrasono-tomography for the heart and great vessels in living human subjects by means of the ultrasonic reflection technique. Jpn Heart J. 1967;8:331-53.

3. Feigenbaum H. Echocardiography. Philadelphia: Lea \& Febiger; 1972.

4. Namekawa KKC, Tsukamoto M. Imaging of blood flow using autocorrelation. Ultrasound Med Biol. 1982;8:138.

5. Matsuo H, Sadogawa H, Ogawa T, et al. Standard method for ultrasound evaluation of deep vein thrombosis and lower extremity varicose veins. Jyoumyakugaku. 2018;29:363-94.

6. Chamsi-Pasha MA, Sengupta PP, Zoghbi WA. Handheld echocardiography. Circulation. 2017;136:2178-88.

7. Pershad J, Myers S, Plouman C, et al. Bedside limited echocardiography by the emergency physician is accurate during evaluation of the critically ill patient. Pediatrics. 2004;114:e667-71.

8. Jensen MB, Sloth E, Larsen KM, et al. Transthoracic echocardiography for cardiopulmonary monitoring in intensive care. Eur J Anaesthesiol. 2004;21:700-7.

9. Manasia AR, Nagaraj HM, Kodali RB, et al. Feasibility and potential clinical utility of goal-directed transthoracic echocardiography performed by noncardiologist intensivists using a small hand-carried device (sonoheart) in critically ill patients. J Cardiothorac Vasc Anesth. 2005;19:155-9.

10. Vignon P, Dugard A, Abraham J, et al. Focused training for goal-oriented hand-held echocardiography performed by noncardiologist residents in the intensive care unit. Int Care Med. 2007;33:1795-9.

11. Gunst M, Sperry J, Ghaemmaghami V, et al. Bedside echocardiographic assessment for trauma/critical care: the beat exam. J Am Coll Surg. 2008;207:e1-3.

12. Cowie B. Focused cardiovascular ultrasound performed by anesthesiologists in the perioperative period: feasible and alters patient management. J Cardiothorac Vasc Anesth. 2009;23:450-6.

13. Ferrada P, Murthi S, Anand RJ, et al. Transthoracic focused rapid echocardiographic examination: real-time evaluation of fluid status in critically ill trauma patients. J Trauma Acute Care Surg. 2011;70:56-64.

14. Takumi I, Hiroshi C, Kayoko A, et al. Diagnosis argorithm of deep vein thrombosis (dvt) medical examination needed at a large-scale disaster. Igaku Kensa. 2017;66:449-62.

15. Jehle D, Davis E, Evans T, et al. Emergency department sonography by emergency physicians. Am J Emerg Med. 1989;7:605-11.

16. Moore CL, Copel JA. Point-of-care ultrasonography. N Engl J Med. 2011;364:749-57.

17. Spencer KT, Kimura BJ, Korcarz CE, et al. Focused cardiac ultrasound: recommendations from the american society of echocardiography. J Am Soc Echocardiogr. 2013;26:567-81.

18. Kimura BJ, Yogo N, O'Connell CW, et al. Cardiopulmonary limited ultrasound examination for "quick-look" bedside application. Am J Cardiol. 2011;108:586-90.

19. Kratz T, Exner M, Campo dell'Orto M, et al. A pocket-sized hand held ultrasound system for intraoperative transthoracic echocardiography by anaesthesiologists: a feasibility study. Technol Health Care. 2016;24:309-15.

20. Breitkreutz R, Uddin S, Steiger H, et al. Focused echocardiography entry level: new concept of a 1-day training course. Minerva Anestesiol. 2009;75:285-92.

21. Breitkreutz R, Price S, Steiger HV, et al. Focused echocardiographic evaluation in life support and peri-resuscitation 
of emergency patients: a prospective trial. Resuscitation. 2010;81:1527-33.

22. Neskovic AN, Hagendorff A, Lancellotti P, et al. Emergency echocardiography: the European Association of Cardiovascular Imaging recommendations. Euro Heart J Cardiovasc Imag. 2013;14:1-11.

23. Labovitz AJ, Noble VE, Bierig M, et al. Focused cardiac ultrasound in the emergent setting: A consensus statement of the American Society of Echocardiography and American College of Emergency Physicians. J Am Soc Echocardiogr. 2010;23:1225-30.

24. American College of Emergency P. Emergency ultrasound guidelines. Ann Emerg Med. 2009;53:550-70.

25. Mayo PH, Beaulieu Y, Doelken P, et al. American college of chest physicians/la société de réanimation de langue française statement on competence in critical care ultrasonography. Chest. 2009;135:1050-60.

26. Neskovic AN, Edvardsen T, Galderisi M, et al. Focus cardiac ultrasound: the European Association of Cardiovascular Imaging viewpoint. Eur Heart J Cardiovasc Imaging. 2014;15:956-60.

27. Via G, Hussain A, Wells M, et al. International evidence-based recommendations for focused cardiac ultrasound. J Am Soc Echocardiogr. 2014;27:683.e1-33.

28. Neskovic AN, Skinner H, Price S, et al. Focus cardiac ultrasound core curriculum and core syllabus of the European Association of Cardiovascular Imaging. Eur Heart J Cardiovasc Imaging. 2018;19:475-81.

29. Perera $P$, Mailhot T, Riley D, et al. The rush exam: rapid ultrasound in shock in the evaluation of the critically ill. Emerg Med Clin N Am. 2010;28:29 vii-56 vii.

30. Ghane MR, Gharib MH, Ebrahimi A, et al. Accuracy of rapid ultrasound in shock (rush) exam for diagnosis of shock in critically ill patients. Trauma Mon. 2015;20:e20095.

31. Nishigami K. Update on cardiovascular echo in aortic aneurysm and dissection. Ann vasc dis. 2018;11:437-42.

32. Blaivas M, Lambert MJ, Harwood RA, et al. Lower-extremity Doppler for deep venous thrombosis-can emergency physicians be accurate and fast? Acad Emerg Med. 2000;7:120-6.

33. Frazee BW, Snoey ER, Levitt A. Emergency department compression ultrasound to diagnose proximal deep vein thrombosis. J Emerg Med. 2001;20:107-12.

34. Theodoro D, Blaivas M, Duggal S, et al. Real-time b-mode ultrasound in the ed saves time in the diagnosis of deep vein thrombosis (DVT). Am J Emerg Med. 2004;22:197-200.
35. Abe Y. Screening for aortic stenosis using physical examination and echocardiography. J Echocardiogr. 2021;19:80-5.

36. Alexander JH, Patel MR. Screening for structural heart disease: time to stop listening and start looking. Am Heart J. 2003;146:570-1.

37. Solomon SD, Saldana F. Point-of-care ultrasound in medical education-stop listening and look. N Engl J Med. 2014;370:1083-5.

38. Zhang L, Wang B, Zhou J, et al. Bedside focused cardiac ultrasound in covid-19 from the Wuhan epicenter: the role of cardiac point-of-care ultrasound, limited transthoracic echocardiography, and critical care echocardiography. J Am Soc Echocardiogr. 2020;33:676-82.

39. Cardim N, Dalen H, Voigt JU, et al. The use of handheld ultrasound devices: a position statement of the European Association of Cardiovascular Imaging (2018 update). Eur Heart J Cardiovasc Imaging. 2019;20:245-52.

40. Kirkpatrick JN, Mitchell C, Taub C, et al. ASE statement on protection of patients and echocardiography service providers during the 2019 novel coronavirus outbreak: endorsed by the American College of Cardiology. J Am Soc Echocardiogr. 2020;33:648-53.

41. Johri AM, Galen B, Kirkpatrick JN, et al. ASE statement on pointof-care ultrasound during the 2019 novel coronavirus pandemic. J Am Soc Echocardiogr. 2020;33:670-3.

42. Doi S, Izumo M, Shiokawa N, et al. Mcconnell's sign assessed by point-of-care cardiac ultrasound associated with in-hospital mortality of covid-19 patients with respiratory failure. J Echocardiogr. 2021;19:67-9.

43. Dweck MR, Bularga A, Hahn RT, et al. Global evaluation of echocardiography in patients with covid-19. Eur Heart J Cardiovasc Imag. 2020;21:949-58.

44. Skulstad H, Cosyns B, Popescu BA, et al. Covid-19 pandemic and cardiac imaging: EACVI recommendations on precautions, indications, prioritization, and protection for patients and healthcare personnel. Eur Heart J Cardiovas Imag. 2020;21:592-8.

Publisher's Note Springer Nature remains neutral with regard to jurisdictional claims in published maps and institutional affiliations. 